\title{
Incidence, Prevalence, and Mortality Rate of Gastrointestinal Cancer in Isfahan, Iran: Application of the MIAMOD Method
} Farhad Moradpour ${ }^{1,7 *}$, Ali Gholami ${ }_{5}^{2}$ Mohammad Salehi , Kamiar Mansori ${ }^{4}$, Mohammad Reza Maracy $_{7}$, Setareh Javanmardi , Abdolhalim Rajabi , Yousef Moradi ${ }^{7}$, Mahmod Khodadost

\begin{abstract}
Gastrointestinal cancers remain the most prevalent cancers in many developing countries such as Iran. The aim of this study was to estimate incidence, prevalence and mortality, as well as time trends for gastrointestinal cancers in Isfahan province of Iran for the period 2001 to 2010 and to project these estimates to the year 2020. Estimates were driven by applying the MIAMOD method (a backward calculation approach using mortality and relative survival rates). Mortality data were obtained from the Ministry of Health and the relative survival rate for all gastrointestinal cancers combined was derived from the Eurocare 3 study. Results indicated that there were clear upward trends in age adjusted incidence (males 22.9 to 74.2 and females 14.9 to 44.2 ), prevalence (males 52.6 to 177.7 and females 38.3 to 111.03), and mortality (males 14.6 to 47.2 and females 9.6 to 28.2) rates per 100,000 for the period of 2001 to 2010 and this upward state would persist for the projected period. For the entire period, the male to female ratio increased slightly for all parameters (incidence rate increased from 1.5 to 1.7 , prevalence from 1.4 to 1.6 , and mortality from 1.5 to 1.7 ). In males, totals of 2,179 incident cases, 5,097 prevalent cases and 1,398 mortality cases were predicated to occur during the study period. For females the predicted figures were 1,379, 3,190 and 891, respectively. It was concluded that the upward trend of incidence alongside increase in survival rates would induce a high burden on the health care infrastructure in the province of Isfahan in the future.
\end{abstract}

Keywords: Gastrointestinal cancer - MIOMED model - incidence - prevalence - mortality - Isfahan, Iran

Asian Pac J Cancer Prev, 17, Cancer Control in Western Asia Special Issue, 11-15

\section{Introduction}

Among different types of cancers, gastrointestinal (GI) tract cancers are the most important ones in terms of their broad spectrum and overall incidence. These cancers account for $15 \%$ of the estimated death worldwide and $20 \%$ of the estimated new cancer cases (Herszenyi and Tulassay, 2010). GI system is the most common organ involved with more than $38 \%$ of all cancers (Sadjadi et al., 2005). After breast and lung cancer, colorectal, stomach, and liver cancers are the major ones (Global Burden of Disease Cancer et al., 2015). In Iran, GI cancers are one of the most important cancers because they account for more than 60 percent of deaths from cancer (Naghibzadeh Tahami et al., 2014). Along with the westernization of the lifestyle and fast economic development, it seems that many developing countries such as Iran have experienced increasing incidence rates of gastrointestinal cancers (Hosseini et al., 2004). Data collected on incidence, prevalence and cancer mortality are the primary resources not only for epidemiological research but also for quantification of the burden of the disease and resource allocation for planning health care interventions. These data can be used to extrapolate the trend of cancer and its variation in the future, and also to estimate requirements for handling the treatment (Jensen and Storm, 1991). Mortality data are gathered systematically at the national level by national organization for civil registration (NOFCR) in collaboration with the Ministry of Health (MOH) in Iran (Jafari et al., 2009). The data including incidence and prevalence were collected and analyzed by population based cancer registries (PBCRs) which cover only a fraction, i.e. $16 \%$, of the population (Etemadi et al., 2008). However, because of

${ }^{1}$ Social Determinants of Health Research Center, Kurdistan University of Medical Sciences, Sanandaj, ${ }^{2}$ Department of Public Health, School of Public Health, Neyshabur University of Medical Sciences, Neyshabur, ${ }^{3}$ Department of Biostatistics, Faculty of Medicine, Shiraz University of Medical Sciences, Shiraz, ${ }^{4}$ Department of Epidemiology, School of Public Health, Shahid Beheshti University of Medical Sciences, ${ }^{7}$ Iran University of Medical Sciences, ${ }^{8}$ Gastroenterology and Liver Research Center, Baqiyatallah University of Medical Sciences, Tehran, ${ }^{5}$ Department of Epidemiology and Biostatistics, Isfahan University of Medical Science, Isfahan, ${ }^{6}$ Department of Anatomical Sciences, Faculty of Nursing and Midwifery, Kermanshah University of Medical Sciences, Kermanshah, Iran *For correspondence: f-moradpour@razi.tums.ac.ir 
data processing, incidence and prevalence information are published with a long delay after initial data collection (Parkin, 2006). In this situation, when it is not possible to obtain accurate statistics on the cancer burden on regional or national level, utilization of the estimation and prediction methods is essential for both cancer research and health care prevention programs (Bray and Moller, 2006).

The aim of this study was to estimate the incidence and prevalence of gastrointestinal cancers in Isfahan province, Iran, based on the mortality and survival data during 2001-2010; also, there was an attempt to predict the incidence, prevalence and mortality until the year 2020 .

\section{Materials and Methods}

To carry out this study, we considered all the mortality data and causes of mortality due to gastrointestinal cancer (International Classification of Disease for Oncology 3rd revision: code C00-C26) from two sources; NOFCR and $\mathrm{MOH}$ death registration systems. Mortality data in Iran are collected from Health Houses and Health Centers in the rural and urban areas, hospitals, forensic medicine and cemeteries to district health centers. In district health center, data are inspected by civil registration. Data are then sent to the provincial health center every 3-6 months and then to the ministry of health annually (Jafari et al., 2009). Population profile by age, sex and calendar year of the period 20012010 were obtained from the statistics center of Iran. There is no study conducted to compute and estimate the relative survival for the digestive tract cancer in Iran. Therefore, we used tabulated relative survival data for the period of diagnosis 1983-1994 of Italian registry obtained from CDROM of EUROCARE-3 study (Roazzi et al., 2003). These data were categorized based on the time of diagnosis to two year interval and following age classes of diagnosis: 15-44, 45-54, 65-74 and 75-99 years.

The MIAMOD method (Verdecchia et al., 1989; De Angelis et al., 1994; Ventura et al., 2012) (Mortality Incidence Analysis Model) was used to derive incidence, prevalence and mortality statistics. It is a back calculation approach starting from mortality and relative survival to estimation and prediction of irreversible diseases such as cancer. Ad hoc MIAMOD/PIAMOD software developed by De Angelis et al (1994) was used for extracting estimation. This method mathematically relates the mortality and prevalence to a putative cancer to incidence and survival probabilities. Join point regression program was used to do a trend analysis, with the aim of observing whether the estimated trends are statistically significant or

Table 1. Gastrointestinal Expected Crude Rates and Expected Cases $\times 100,000$ by Sex and Calendar Year in Isfahan Province, Iran

\begin{tabular}{|c|c|c|c|c|c|c|c|c|c|c|c|c|}
\hline \multirow[b]{3}{*}{$\begin{array}{l}\text { Calendar } \\
\text { year }\end{array}$} & \multicolumn{4}{|c|}{ Mortality } & \multicolumn{4}{|c|}{ Incidence } & \multicolumn{4}{|c|}{ Prevalence } \\
\hline & \multicolumn{2}{|c|}{ Male } & \multicolumn{2}{|c|}{ female } & \multicolumn{2}{|c|}{ Male } & \multicolumn{2}{|c|}{ female } & \multicolumn{2}{|c|}{ Male } & \multicolumn{2}{|c|}{ female } \\
\hline & $\begin{array}{c}0-99 \\
\text { Crud rate }\end{array}$ & $\begin{array}{l}0-99 \\
\text { cases }\end{array}$ & $\begin{array}{c}0-99 \\
\text { Crud } \\
\text { rate }\end{array}$ & $\begin{array}{l}0-99 \\
\text { cases }\end{array}$ & $\begin{array}{c}0-99 \\
\text { Crud } \\
\text { rate }\end{array}$ & $\begin{array}{l}0-99 \\
\text { cases }\end{array}$ & $\begin{array}{c}0-99 \\
\text { Crud } \\
\text { rate }\end{array}$ & $\begin{array}{l}0-99 \\
\text { cases }\end{array}$ & $\begin{array}{l}\text { 0-99 } \\
\text { Crud } \\
\text { rate }\end{array}$ & $\begin{array}{l}0-99 \\
\text { cases }\end{array}$ & $\begin{array}{c}0-99 \\
\text { Crud } \\
\text { rate }\end{array}$ & $\begin{array}{l}0-99 \\
\text { cases }\end{array}$ \\
\hline 2001 & 12.3 & 268.0 & 8.7 & 181.0 & 19.2 & 417.0 & 13.4 & 279.0 & 44.1 & 956.0 & 33.2 & 688.0 \\
\hline 2002 & 13.1 & 289.0 & 9.2 & 193.0 & 20.5 & 450.0 & 14.2 & 298.0 & 47.2 & 1040.0 & 34.9 & 735.0 \\
\hline 2003 & 13.9 & 310.0 & 9.7 & 206.0 & 21.7 & 483.0 & 15.0 & 318.0 & 50.4 & 1122.0 & 36.9 & 784.0 \\
\hline 2004 & 14.9 & 334.0 & 10.2 & 220.0 & 23.2 & 522.0 & 15.8 & 339.0 & 54.2 & 1217.0 & 38.9 & 835.0 \\
\hline 2005 & 15.9 & 360.0 & 10.8 & 234.0 & 24.8 & 561.0 & 16.7 & 362.0 & 58.0 & 1315.0 & 41.1 & 891.0 \\
\hline 2006 & 16.9 & 395.0 & 11.4 & 254.0 & 26.4 & 616.0 & 17.6 & 392.0 & 62.0 & 1447.0 & 43.4 & 964.0 \\
\hline 2007 & 18.0 & 424.0 & 12.1 & 271.0 & 28.0 & 662.0 & 18.7 & 419.0 & 66.0 & 1561.0 & 46.0 & 1031.0 \\
\hline 2008 & 19.2 & 455.0 & 13.0 & 292.0 & 29.9 & 710.0 & 20.0 & 452.0 & 70.7 & 1677.0 & 49.2 & 1109.0 \\
\hline 2009 & 20.5 & 488.0 & 13.8 & 312.0 & 31.9 & 760.0 & 21.3 & 482.0 & 75.5 & 1800.0 & 52.2 & 1184.0 \\
\hline 2010 & 21.9 & 524.0 & 14.7 & 335.0 & 34.1 & 816.0 & 22.6 & 517.0 & 80.9 & 1938.0 & 55.6 & 1269.0 \\
\hline 2011 & 24.1 & 583.0 & 16.2 & 375.0 & 37.5 & 908.0 & 25.0 & 579.0 & 88.6 & 2147.0 & 60.5 & 1398.0 \\
\hline 2012 & 26.4 & 645.0 & 17.8 & 416.0 & 41.0 & 1004.0 & 27.5 & 643.0 & 96.8 & 2369.0 & 65.6 & 1532.0 \\
\hline 2013 & 28.9 & 713.0 & 19.5 & 461.0 & 44.9 & 1111.0 & 30.1 & 712.0 & 105.7 & 2614.0 & 71.2 & 1682.0 \\
\hline 2014 & 31.6 & 789.0 & 21.4 & 511.0 & 49.2 & 1227.0 & 33.0 & 788.0 & 115.5 & 2883.0 & 77.3 & 1846.0 \\
\hline 2015 & 34.5 & 869.0 & 23.3 & 562.0 & 53.6 & 1351.0 & 35.8 & 865.0 & 125.8 & 3169.0 & 83.5 & 2018.0 \\
\hline 2016 & 37.6 & 957.0 & 25.4 & 619.0 & 58.6 & 1491.0 & 39.2 & 956.0 & 137.3 & 3491.0 & 90.9 & 2218.0 \\
\hline 2017 & 41.0 & 1052.0 & 27.6 & 679.0 & 63.9 & 1640.0 & 42.6 & 1051.0 & 149.5 & 3836.0 & 98.6 & 2430.0 \\
\hline 2018 & 44.7 & 1156.0 & 29.9 & 744.0 & 69.7 & 1804.0 & 46.3 & 1153.0 & 163.0 & 4218.0 & 107.0 & 2663.0 \\
\hline 2019 & 48.8 & 1272.0 & 32.5 & 816.0 & 76.0 & 1984.0 & 50.3 & 1263.0 & 177.8 & 4640.0 & 116.2 & 2919.0 \\
\hline 2020 & 53.1 & 1398.0 & 35.2 & 891.0 & 82.8 & 2179.0 & 54.3 & 1378.0 & 193.7 & 5098.0 & 125.8 & 3190.0 \\
\hline
\end{tabular}


Table 2. Average Annual Percentage Change of Incidence (IAAPC) Prevalence (PAAPC) and Mortality (MAAPC) of Gastrointestinal Cancer

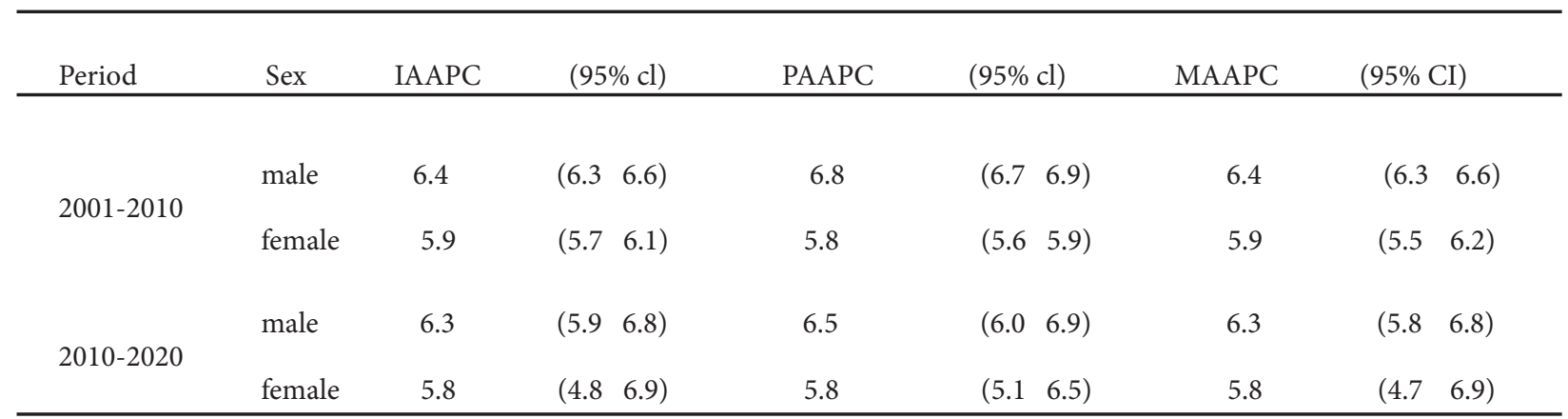

not. The annual variation of the rates has been measured by average annual percentage change (AAPC).

The incidence rate was modeled based on polynomial logistic model as the function of age, period and cohort covariate. The regression coefficients of incidence model were obtained by maximum likelihood method, assuming poison distribution of cancer mortality data (Verdecchia et al., 1989; De Angelis et al., 1994). Incidence was extrapolated to the future based on the period linear trend from 2001-2010, assuming that age and cohort effect do not change over the entire projection period.

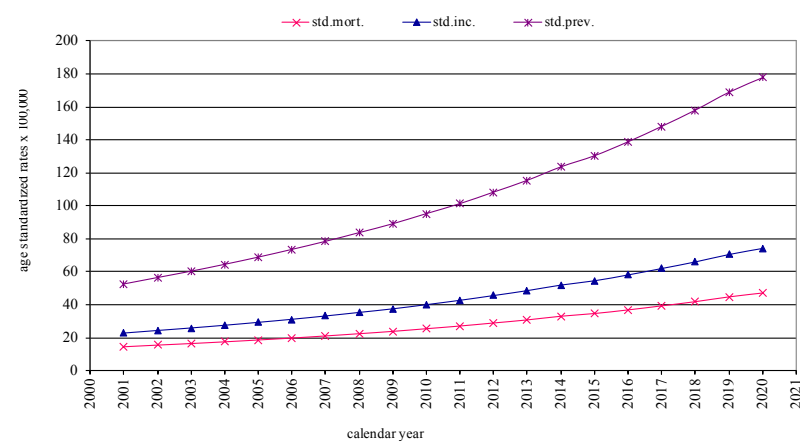

Figure 1. Time Trends of Standard Mortality (std.mort), Incidence (std.inc) and Prevalence (std.prev) Estimates in Male in Isfahan, Iran. Age Standardized Rates (World Population) Per 100,000, Age 0-99 Years

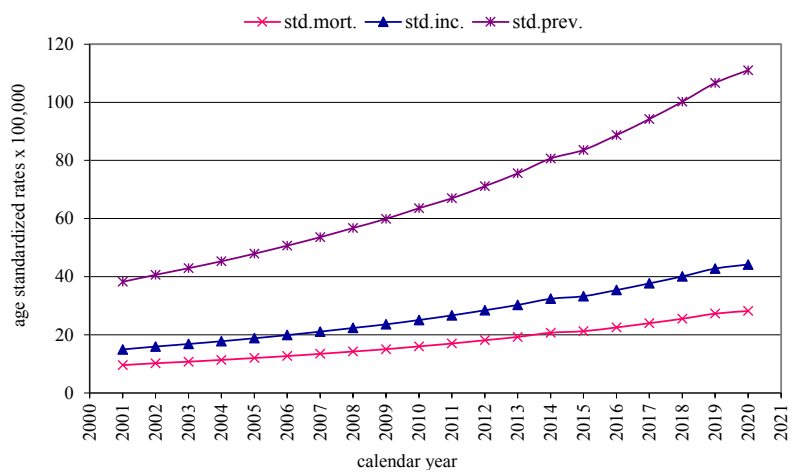

Figure 2. Time Trends of Standard Mortality (std.mort), Incidence (std.inc) and Prevalence (std.prev) Estimates in Female in Isfahan, Iran. Age Standardized Rates (World Population) Per 100,000, Age 0-99 years
The entire estimate referred to the 0-99 year old age class. The age standardized rate was estimated based on the world reference population.

\section{Results}

Incidence, prevalence and mortality prediction of the gastrointestinal cancer until 2020 based on the period 2001-2010 display the same increasing trend for men and women. In men, there is an expected increase in the incidence with age standardized rate (ASR) from 22.9 (crude rate, $\mathrm{CR}=19.2)$ to $74.2(\mathrm{CR}=82.8)$ cases per 100,000 persons year (PY). The prevalence rate would increase from ASR 52.6 (CR=45.1) to 177.7 (193.7) and mortality from ASR 14.6 $(\mathrm{CR}=12.3)$ to 47.2 (53.1) when comparing the year 2001 and 2020 (Figure 1). There is the same incremental trend for women with respect to the incidence rate from ASR $14.9(\mathrm{CR}=13.4)$ to 44.2 $(\mathrm{CR}=54.3)$, prevalence rate from ASR $38.3(\mathrm{CR}=33.2)$ to $111.03(\mathrm{CR}=125.8)$ and mortality from ASR 9.6 $(\mathrm{CR}=8.7)$ to $28.2(\mathrm{CR}=35.2)$ cases per 100,000 person years (Figure 2). Table 1 presents the estimated cases and crud rate for the study period by sex and calendar year. It is estimated that the number of dead cases will increase from 448 to 2,289 by 2,020 ; incidence will increase from 696 to 3,557 and prevalence from 1,644 to 8,287.

Cumulative incidence risk by birth cohort was estimated to increase from 0.3 percent for birth cohort 1,902 to 48 in 1999 in males and it will increase from 0.2 to 24 percent in females (Figure 3 ).

Join point regression demonstrates an average annual percentage change (AAPC) for aforementioned

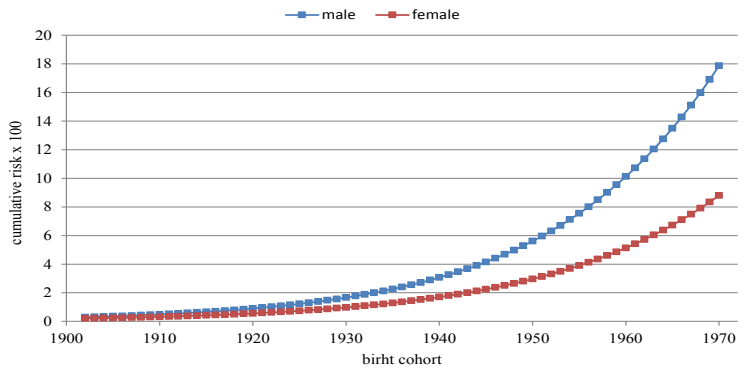

Figure 3. Estimated Cumulative Risk of All Gasterointestinal Cancer Combined by Birth Cohort, Age 0-74 by Gender 
age adjusted rates. Table 2 presents an AAPC and corresponding 95 percent confidence interval for males and females in the period 2001 to 2010 and project period 2010-2020.

\section{Discussion}

It was the main purpose of this study to draw out the updated gastrointestinal cancer burden by means of incidence, prevalence and mortality until 2,022. There are different methods used for estimation of cancer indicators and there is no statistically significant difference between them (Dyba and Hakulinen, 2000). The most obvious finding of this study was that all the trends of cancer indicator have clear upward trends in both males and females for the entire period of the study until 2,022. These trends are consistent with our former estimation for all cancers combined except for non-melanoma skin cancer (Maracy et al., 2012). The estimation of the incidence adjusted rate has increased by $5.9 \%$ and mortality by $5.8 \%$ during 2001 and 2020 . These measures increased by $6.4 \%$ for males, indicating that there is no substantial difference in sex related risk factors. This similar trend by sex was seen overall in Switzerland but unlike the present study the incremental patterns are in the slightest steep (Joliat et al., 2015). In the studies conducted in western countries (Grande et al., 2007; Inghelmann et al., 2007; Budroni et al., 2013; Foschi et al., 2013; Rashid et al., 2013; Rossi et al., 2013), only gastric cancer (the leading cancer in Iran) and relatively female colorectal patterns show a declining manner while in the present study the stomach cancer was found to have a $33 \%$ incidence and $37 \%$ mortality of digestive cancers and as previously estimated it has a strong upward trend (Moradpour and Fatemi, 2013).

In the present study, the incidence and mortality (ASR) point estimation was 45.6 and 28.9 in males, and 28.5 and 18.2 in females, respectively in 2012 ( Figures 1,2). The 2012 Globocan estimation for the incidence and mortality by sex for Iranian population displayed 50.4 and 39.4 per $100,000 \mathrm{PY}$ in males, and 35.9 and 28 per 100,000 PY in females (Ferlay et al., 2012). This means that the residents of Isfahan have common risk factors with those of other parts of Iran, which might resemble in terms of intensity and duration of exposure. Lower death rate might be due to the more survival probability of the patients diagnosed in Isfahan. However, some of these differences might be related to the different procedures used to extract the estimation.

Despite the rapid incremental trend of the digestive system cancers, incidence and mortality showed a slowly diverging pattern over the study period. Such trends account for improvement in the survival of the cancers, which may be due to development of an early detection technique and new therapeutic procedures (De Vries and Kuipers, 2007).

Figure 3 displays a cumulative life time risk up to 74 years old, which is an incidence estimate of all ages during life. The cumulative risk increased more for recent cohort than the older one. This increased risk was more tangible for men (about twice a woman) from the cohort 1940 onwards. Generally, this increased risk might account for great changes in the lifestyle such as high calorie foods intake, lack of physical activity, smoking habits, socio economic factors, and population aging (Lieberman, 2003; Rodgers et al., 2004; Stuckler, 2008).

In 2020, there will be 3,557 new digestive cancer cases that need to be treated for the first time and 2289 cases will die, so it requires to stop medical cares. Approximately, 15,699 patients with gastrointestinal cancer will be diagnosed up to 2020, and therefore, the health care system will be faced with a highly demanded resource.

To conclude, our findings suggest that there is no reduction or leveling off in gastrointestinal cancer incidence in the near future. This incremental trend of incidence alongside the increase in the survival probability can induce a high burden of patient to the population. Finally, because of the existence of different types of socio-economic, behavioral and environmental risk factors in a different geographic area, extensive research is also needed to determine the cancer status at other regions of Iran in the future years. The authorities can use this information for planning heath care and allocating resources in public health.

\section{Acknowledgment}

The authors would like to appreciate the department of Epidemiology and Biostatistics, faculty of health, Isfahan University of Medical Sciences, and disease control center, deputy of health staff for their support and contribution to this study and to thank the Research Consultation Centre (RCC) of Shiraz University of Medical Sciences for editing of the manuscript.

\section{Refrences}

Bray F, Moller B (2006). Predicting the future burden of cancer. Nat Rev Cancer, 6, 63-74.

Budroni M, Sechi O, Cossu A, et al (2013). Estimates of cancer burden in Sardinia. Tumori, 99, 408-15.

De Angelis G, De Angelis R, Frova L, et al (1994). MIAMOD: a computer package to estimate chronic disease morbidity using mortality and survival data. Comput Methods Programs Biomed, 44, 99-107.

De Vries AC, Kuipers EJ (2007). Review article: Helicobacter pylori eradication for the prevention of gastric cancer. Aliment Pharmacol Ther, 26, 25-35.

Dyba T, Hakulinen T (2000). Comparison of different approaches to incidence prediction based on simple interpolation techniques. Stat Med, 19, 1741-52.

Etemadi A, Sadjadi A, Semnani S, et al (2008). Cancer registry in Iran: a brief overview. Arch Iran Med, 11, 577-80.

Ferlay J, Soerjomataram I, Ervik M, et al (2012). GLOBOCAN 2012 v1.0, Cancer Incidence and Mortality world wide: IRAC Cancer base NO.11 online. Available: http://globocan.iarc.fr/Pages/summary table site sel.aspx Accessed 10.292015.

Foschi R, Viviano L, Rossi S (2013). Estimates of cancer burden in Abruzzo and Molise. Tumori, 99, 366-73.

Fitzmaurice C, Dicker D, et al (2015). The Global Burden of Cancer 2013. JAMA Oncol, 1, 505-27.

Grande E, Inghelmann R, Francisci S, et al (2007). Regional estimates of colorectal cancer burden in Italy. Tumori, 93, $352-9$.

Herszenyi L, Tulassay Z (2010). Epidemiology of gastrointestinal 
and liver tumors. Eur Rev Med Pharmacol Sci, 14, 249-58.

Hosseini SV, Izadpanah A, Yarmohammadi H (2004). Epidemiological changes in colorectal cancer in Shiraz, Iran: 1980-2000. Anz J Surg, 74, 547-9.

Inghelmann R, Grande E, Francisci S, et al (2007). Regional estimates of stomach cancer burden in Italy. Tumori, 93, 367-73.

Jafari N, Kabir M, Motlagh M (2009). Death registration system in I.R.Iran. Iran J Public Health, 38, 127-9.

Jensen OM, Storm HH (1991). Cancer registration: principles and methods. Reporting of results. IARC Sci Publ, 108-25.

Joliat GR, Hahnloser D, Demartines N, et al (2015). Future development of gastrointestinal cancer incidence and mortality rates in Switzerland: a tumour registry- and population-based projection up to 2030. Swiss Med Wkly, 145, 141-88.

Lieberman LS (2003). Dietary, evolutionary and modernizing influences on the prevalence of type 2 diabetes. Annu Rev Nutr, 23, 345-77.

Maracy MR, Moradpour F, Hosseini SM, et al (2012). Cancer incidence and prevalence in isfahan: application of mortality data to estimates and projects for the period 2001-2015. Int J Prev Med, 3, 867-74.

Moradpour F, Fatemi Z (2013). Estimation of the projections of the incidence rates, mortality and prevalence due to common cancer site in Isfahan, Iran. Asian Pac J Cancer Prev, 14, 3581-5.

Naghibzadeh Tahami A, Khanjani N, Yazdi Feyzabadi V, et al (2014). Opium as a risk factor for upper gastrointestinal cancers: a population-based case-control study in Iran. Arch Iran Med, 17, 2-6.

Parkin DM (2006). The evolution of the population-based cancer registry. Nat Rev Cancer, 6, 603-12.

Rashid I, Pannozzo F, Rossi S, et al (2013). Estimates of cancer burden in Lazio. Tumori, 99, 359-65.

Roazzi P, Capocaccia R, Santaquilani M, et al (2003). Electronic availability of EUROCARE-3 data: a tool for further analysis. Ann Oncol, 14, 150-5.

Rodgers A, Ezzati M, Vander Hoorn S, et al (2004). Distribution of major health risks: findings from the Global Burden of Disease study. PLoS Med, 1, 27.

Rossi S, Crocetti E, Capocaccia R, et al (2013). Estimates of cancer burden in Italy. Tumori, 99, 416-24.

Sadjadi A, Nouraie M, Mohagheghi MA, et al (2005). Cancer occurrence in Iran in 2002, an international perspective. Asian Pac J Cancer Prev, 6, 359-63.

Stuckler D (2008). Population causes and consequences of leading chronic diseases: a comparative analysis of prevailing explanations. Milbank Q, 86, 273-326.

Ventura L, Miccinesi G, Sacchettini C, et al (2012). Estimating all cancers incidence with the MIAMOD model: a new method to include multiple tumors. Tumori, 98, 296-302.

Verdecchia A, Capocaccia R, Egidi V, et al (1989). A method for the estimation of chronic disease morbidity and trends from mortality data. Stat Med, 8, 201-16. 